\title{
The effect of noise on the human body, in particular, on cardiovascular diseases
}

\author{
Elena Staseva $^{1}$, Marina Kvitkina $^{1}$, Artyom Litvinov ${ }^{2}$, Nataliya Kobzeva ${ }^{3, *}$ \\ ${ }^{1}$ Don State Technical University, 1, pl. Gagarina, 344002, Rostov-on-Don, Russia \\ ${ }^{2}$ Kuban State Technological University, 2, str. Moscow, 350072, Krasnodar, Russia \\ ${ }^{3}$ Rostov State Medical University, 29, Nakhichevan lane, 344022, Rostov-on-Don, Russia
}

\begin{abstract}
Some manufacturing processes are accompanied by significant noise and vibration. Sources of intense noise and vibration are machines and mechanisms with unbalanced rotating masses, as well as technological installations and apparatuses in which the movement of gases and liquids occurs at high speeds and has a pulsating character. The modern development of technology, equipping enterprises with powerful and fastmoving machines and mechanisms leads to the fact that people are constantly exposed to noise of increasing intensity. Increasing noise and vibration in the workplace has a harmful effect on the human body. As a result of prolonged exposure to noise, the normal activity of the cardiovascular and nervous systems, digestive and hematopoietic organs is disturbed, and professional hearing loss develops, the progression of which can lead to complete hearing loss. Among industrial hazards, noise and vibration occupy one of the leading places among production hazards. The harmful effects of increased noise levels on the human body are well khown, therefore, the relevance of this problem is obvious.
\end{abstract}

\section{Introduction}

In production conditions, a variety of machines, apparatus and tools are sources of noise, vibration.

Noise and vibration are mechanical vibrations propagating in gaseous and solid media. Noise and vibration differ in frequency of oscillations.

Noise is an erratic combination of sounds of different strength and frequency; able to have an adverse effect on the body. The source of noise is any process that causes local pressure changes or mechanical vibrations in harsh, watery or gaseous media. Sources of noise can be engines, pumps, compressors, turbines, pneumatic and electric tools, hammers, threshers, machine tools, centrifuges, silos and other installations that have moving parts. In addition, in recent years, due to the significant development of urban transport, the noise intensity in everyday life has also increased, because he has acquired an enormous social significance as an unfavorable factor $[1,3,5,7]$.

Vibration is small mechanical vibrations that occur in elastic bodies under the influence of variable forces.

*Corresponding author: 5976765@mail.ru 
When working in conditions of vibration, labor productivity decreases, the number of injuries increases. At some workplaces in agricultural production, vibrations exceed normalized values, and in some cases they are close to limit. The vibration levels on the controls do not always comply with the standards. Typically, low-frequency vibrations that negatively affect the body predominate in the vibration spectrum. Some types of vibration adversely affect the nervous and cardiovascular systems, the vestibular apparatus. The most harmful effect on the human body is provided by vibration, the frequency of which coincides with the frequency of natural vibrations of individual organs, the approximate values of which are as follows $(\mathrm{Hz})$ : stomach - $2 \ldots 3$; kidneys - $6 \ldots 8$; heart - 4 ... 6; intestines $-2 \ldots 4$; vestibular apparatus - 0.5..L, 3; eyes - $40 \ldots 100$, etc.

The effect on muscular reflexes reaches $20 \mathrm{~Hz}$; the tractor-loaded seat on the tractor has its own vibration frequency of $1.5 \ldots 1.8 \mathrm{~Hz}$, and the rear wheels of the tractor - $4 \mathrm{~Hz}$. The vibration is transmitted to the human body at the moment of contact with the vibrating object: when acting on the limbs, local vibration occurs, and on the whole body - general. Local vibration affects neuromuscular tissues and the musculoskeletal system and leads to spasms of peripheral vessels. With prolonged and intense vibrations, in some cases, a professional pathology develops (local vibration often leads to it): peripheral, cerebral or cerebral-peripheral vibrational disease. In the latter case, there are changes in cardiac activity, general excitement, or, conversely, inhibition, fatigue, the appearance of pain, a feeling of shaking of the internal organs, nausea. In these cases, vibrations also affect the osteoarticular apparatus, muscles, peripheral circulation, vision, hearing $[1,3,5,7,9]$. Local vibrations cause spasms of blood vessels that develop from the terminal phalanges of the fingers, spreading to the entire hand, forearm, and cover the vessels of the heart.

Workers can be exposed to occupational vibration through the use of power or pneumatic hand tools or other machinery, or by driving large transportation, construction or agricultural vehicles. Vibration that is generated through the use of powered hand tools, and is transmitted from the tool to the hand-arm system is referred to as hand-transmitted vibration. However, recent studies have also demonstrated that vibration can be transmitted through platforms workers are standing on, and in these situations, the point of contact is the feet. Workers can also be exposed to whole body vibration (WBV). WBV exposure occurs in occupations where workers are driving trucks, large earth moving vehicles, or where they are using hand tools where the amplitude of the vibration is great enough to be transmitted to other portions of the body, such as in workers using jack-hammers.

Exposure to WBV is of concern within the workforce because it's associated with the development of a number of negative health consequences including back and neck pain, and potentially, cardiovascular disease, the development of various neuropathies, digestive problems, headaches, dizziness, motion sickness and possibly cancer. However, workers exposed to WBV are often also exposed to a number of other risk factors that may contribute to the development of these negative health effects. These risk factors include maintaining a static posture for a long-period of time, torque or twisting of the abdomen to view the area around the vehicle, and heavy lifting that often occurs when a vehicle is being loaded or unloaded. In addition to vibration and the physical exposures associated with a job, there may be other co-exposures to chemicals or certain environmental conditions that contribute to the development of disease or injury in workers. Because most workers are exposed to multiple factors that may induced injury or illness, it's difficult to determine which factors pose the greatest risk for inducing injury or illness. Experimental studies, examining the effects of each of these factor individually on health outcomes can provide additional information that will help determine the contribution of each exposure factor to various health problems.

This review will describe the industrial sectors where vibration exposure is most prevalent and the health effects associated with exposure to HTV and WBV. Experimental 
methods used to measure and characterize vibration generated in various occupational settings are discussed, and models that have been used to uncover the etiology of vibrationinduced injuries. Although numerous studies have been published on both HTV and HBV, there are new epidemiological studies showing an increased risk of specific cancers with exposure to WBV. Therefore additional etiological studies need to be performed. New avenues for research are discussed below.

\section{Methods}

Noise is one of the most common adverse physical causes of the environment, acquiring fundamental socio-hygienic importance, in connection with urbanization, as well as the mechanization and automation of technological processes, the upcoming development of diesel engineering, jet aircraft, and transport. For example, when starting jet engines of aircraft, the noise level ranges from 120 to $140 \mathrm{~dB}$ with riveting and chopping of sheet steel - from 118 to $130 \mathrm{~dB}$, the work of woodworking machines - from 100 to $120 \mathrm{~dB}$, weaving machines - up to $105 \mathrm{~dB}$; household noise associated with the life of people is $45-60 \mathrm{~dB}$.

For hygienic assessment, noise is divided into:

- by the nature of the range - broadband with a continuous range of a width of more than one octave and tonal, in the range of which there are discrete tones;

- in terms of spectral composition - to low-frequency (maximum sound energy falls at frequencies below $400 \mathrm{~Hz}$ ), mid-frequency (maximum sound energy at frequencies from 400 to $1000 \mathrm{~Hz}$ ) and frequency (maximum sound energy at frequencies above $1000 \mathrm{~Hz}$ );

- in terms of time - unchanged (the sound level changes in time but most than $5 \mathrm{~dB}$ - on a scale A) and unstable.

One of the main sources of noise in the city is road transport, the traffic intensity of which is constantly growing. The highest noise levels of 90-95 dB are observed on the main streets of cities with an average traffic intensity of 2-3 thousand or more transport units per hour. The level of street noise is determined by the intensity, speed and nature (composition) of the traffic flow. In addition, it depends on planning decisions (longitudinal and transverse street profiles, building height and density) and improvement elements such as roadway coverage and the presence of green spaces. Each of these factors can change the level of transport noise within up to $10 \mathrm{~dB}$. In an industrial city, the percentage of freight transport on highways is usually high. An increase in the total flow of trucks, especially heavy trucks with diesel engines, leads to an increase in noise levels. In general, trucks and cars create heavy noise in urban areas. The noise arising on the roadway of the highway extends not only to the main road, but also deep into the housing estate. So, in the zone of the most severe noise exposure are parts of quarters and microdistricts located along highways of citywide significance (equivalent noise levels from 67.4 to $76.8 \mathrm{~dB}$ ). Noise levels measured in living rooms with open windows oriented to the indicated highways are only 10-15 dB lower. The acoustic characteristic of the traffic flow is determined by the noise figures of cars. The noise produced by individual transport crews depends on many factors: engine power and operating mode, crew technical condition, road surface quality, and speed. In addition, the noise level, as well as the efficiency of car operation, depends on the qualifications of the driver. The noise from the engine increases sharply when it starts and warms up (up to $10 \mathrm{~dB}$ ). The movement of the car at the first speed (up to $40 \mathrm{~km} / \mathrm{h}$ ) causes excessive fuel consumption, while the engine noise is 2 times higher than the noise created by it at the second speed. Significant noise causes a sharp braking of the car when driving at high speed. Noise is noticeably reduced if the speed is suppressed due to engine braking until the foot brake is applied. Recently, the average noise level produced by 
vehicles has increased by $12-14 \mathrm{~dB}$. That is why the problem of noise control in the city is becoming increasingly acute.

The human response to noise is different. Some people are tolerant of noise, while others cause irritation, the desire to get away from the source of noise. The psychological assessment of noise is mainly based on the concept of perception, with internal tuning to the noise source being of great importance. It determines whether the noise will be perceived as disturbing. Often the noise reproduced by the person himself does not bother him, while the small noise caused by neighbors or some other source has a strong annoying effect $[2,4,6,8]$.

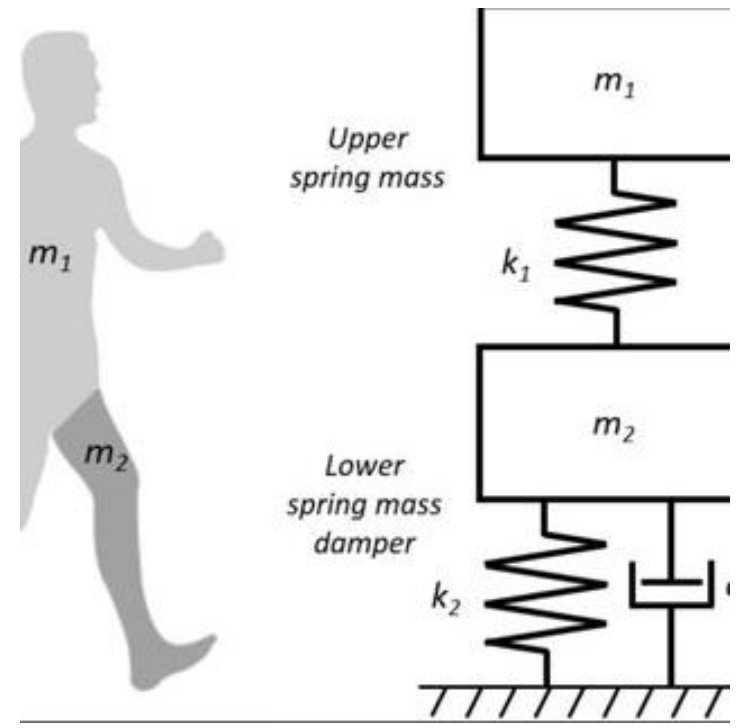

Fig. 1. Vibration effects.

In conditions of strong city noise, a constant voltage of the auditory analyzer occurs. This causes an increase in the threshold of hearing $(10 \mathrm{~dB}$ for most people with normal hearing) by 10-25 dB. Noise makes speech intelligibility difficult, especially at a level of more than $70 \mathrm{~dB}$. The damage caused to the hearing by loud noise depends on the spectrum of sound vibrations and the nature of their change. The danger of possible hearing loss due to noise largely depends on the individual characteristics of the person. Some lose their hearing even after a short exposure to noise of relatively moderate intensity, while others can work with strong noise for almost their entire lives without any noticeable hearing loss. Constant exposure to loud noise can not only adversely affect hearing, but can also cause other harmful effects.

\section{Results}

One of the areas of noise control is the development of state standards for vehicles, engineering equipment, household appliances, which are based on hygiene requirements to ensure acoustic comfort. GOST 19358-85 "External and internal noise of vehicles. Permissible levels and measurement methods "establishes noise characteristics, methods for their measurement and acceptable noise levels of cars (motorcycles) of all samples accepted for state, interdepartmental, departmental and periodic control tests. As the main characteristic of external noise, the sound level is adopted, which should not exceed 85-92 $\mathrm{dB}$ for cars and buses, and 80-86 dB for motorcycles. Approximate values of permissible sound pressure levels in octave frequency bands are given for internal noise: sound levels 
are $80 \mathrm{~dB}$ for cars, cabs or workplaces of truck drivers, buses - $85 \mathrm{~dB}$, passenger bus areas $75-80 \mathrm{~dB}[9,10,11]$.

Intense noise emission is created by the frame and flywheel of a mechanical press. The vibrations of the bed and the flywheel are excited as a result of impacts in all movable joints of the press that occur at the moment of switching on and at the beginning of the movement of the crank or eccentric mechanism, that is, when the gaps in the joints of the connecting rod with the working shaft neck and slider are sampled bearings of the working shaft. The process of interaction between the stamp and the workpiece also has an impact character. The increase in stamping noise levels reaches $10 \mathrm{dBA}$. There is no noise of turning on the press during automatic operation. Noise levels do not change compared to the single start mode. Noise reduction can be achieved by ensuring a smooth operation of the switching process, which is realized by replacing mechanical cam couplings with friction or pneumatic ones. Noise during stamping is reduced when using beveled dies, instead of straight ones, which contributes to a smooth working process. These measures allow to reduce noise at the workplace of the puncher by $14 \mathrm{dBA}$. The use of such dies is justified when cutting parts of a large perimeter.

Preliminary operations (rolling, broaching, draft) of stamping on hammers are advisable to perform on separate equipment (forging rollers, hammers), since when performing preliminary and final stamping on a single hammer, strikes with different energies are applied, which requires a highly skilled blacksmith when controlling a hammer. The use of software control of the energy of the hammer strike eliminates the possibility of a collision of the mirror stamps during the preliminary operations.

Studies have established that stamp vibrations are transmitted through the die holder and rammers to the racks and the hammer cylinder and, in addition, during eccentric impacts through the head and guides, also to the hammer racks.

Sanitary norms of permissible noise necessitate the development of technical, architectural, planning and administrative measures aimed at creating a noise regime that meets hygienic requirements, both in urban buildings and in buildings for various purposes, which help to maintain the health and working capacity of the population. One of the effective means of combating industrial noise is the use of damping metallic and nonmetallic materials. However, non-metals are not used to reduce impact noise due to their low strength characteristics, and metal materials with high strength properties provide very little noise reduction, so the question arose of creating fundamentally new materials that could have high strength characteristics and sufficient damping properties [12]. Such materials are bimetals, which make it possible to obtain a combination of service properties that cannot be obtained in a single metal or alloy, for example: high strength with corrosion resistance, impact strength with wear resistance, strength with high electrical and thermal conductivity, high strength and sufficient damping properties etc. Until now, timid attempts to use bimetals to reduce noise and vibration have not provided a solution to the problem, therefore, a scientific study on the development of bimetals with enhanced damping properties is very relevant [13]. Technical means of noise protection: sound absorption, sound insulation, shielding, damping and noise mufflers. Personal protective equipment.

Noise control measures:

Replacement of noisy processes by noiseless or less noisy;

Improving the quality of manufacturing and installation of equipment;

Shelter of noise sources;

The withdrawal of working people from the sphere of noise;

the use of personal protective equipment. 


\section{Discussion}

The influence of noise and vibration on a person and his body in recent decades has become one of the most urgent problems in all countries of the world. Noise affects a person in production (meaning industrial enterprises and some noise objects), the street and in the house.

From an unsatisfactory state of affairs with life safety, the country annually suffers large human, financial, economic, material and moral losses. Ensuring the safety of production and labor protection of workers is one of the main problems of the national security of the country. At present, in our country, many enterprises do not observe safety precautions, and working conditions cannot be called favorable(fig.2).

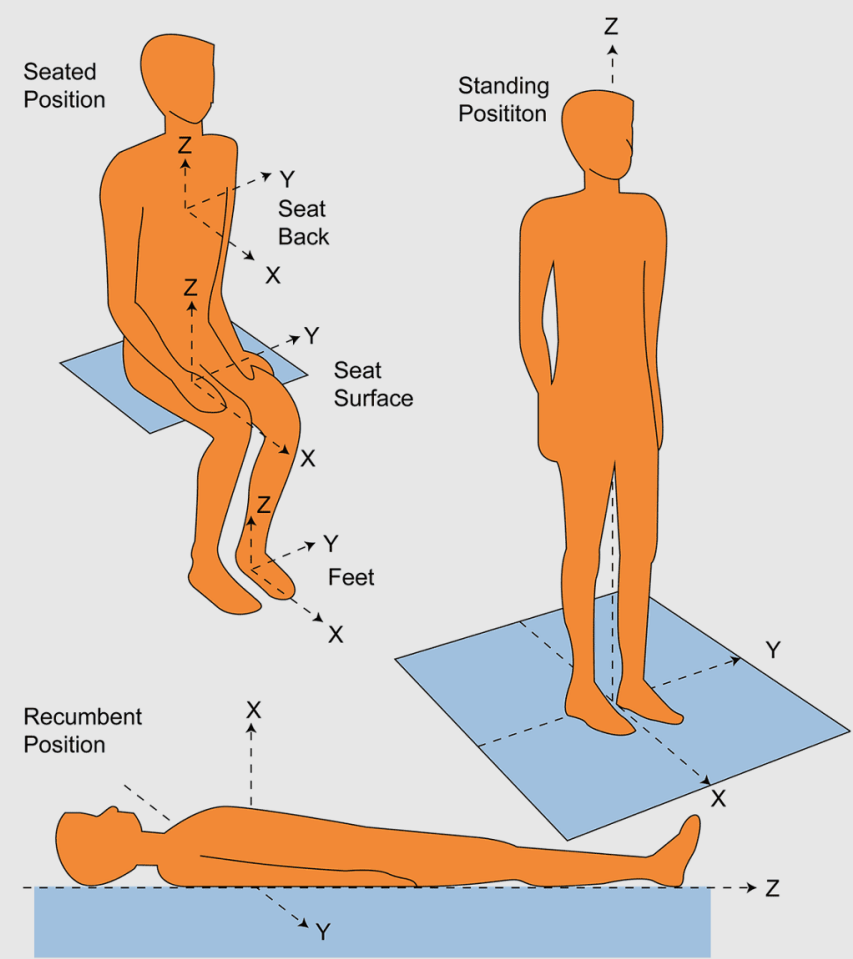

Fig. 2. Vibration effects.

The structural damping of the bed can be increased by closing the rack profile by attaching screens with a sound-absorbing coating (for example, from a layer of mineral wool) on it and installing partitions inside the closed cavities with filling the cells between the partitions with sand, gravel or shot.

To combat noise and vibration, both general and individual protective equipment is used. When planning production facilities, such as an engine test station, thermal and 
blacksmith shops are located on the leeward side in relation to other buildings and a residential area.

To attenuate the noise penetrating outward, it is necessary to use sound insulation of building envelopes. The rationalization of technological processes, the use of silencers, the careful fit of all moving parts of mechanisms - all this many times reduces noise. The greatest effect is achieved by replacing noisy jobs with less noisy ones.

Pneumatic riveting of frames and other parts must be replaced by hydraulic riveting or welding, forging and stamping by pressing.

To reduce vibration, it is also necessary to use special sound-absorbing structures near sources of noise or a workplace, enclose noisy units of the unit in insulating covers (gear reducers, chain, belt and other gears, impact parts, engines). When processing metal rods on automatic turret machines, the safety tube must be spring loaded or a rubber jacket inserted in the hole of this tube.

In order to reduce vibration, it is recommended to use a stiff, spring-free seat in cars, as it is a good vibration damper. Vibration acts on a person through the back, pelvis, arms. Long-term operation of the car leads to fluctuations in body parts, which negatively affects the driver. To reduce vibration, machines should be installed on a foundation deepened below the foundation of the walls, insulated from the soil by air gaps, or on specially designed shock absorbers made of steel springs or made of elastic materials.

To weaken the transmission of vibrations and noise through air ducts and pipelines, they must be connected to the fans and pumps using a flexible insert made of rubberized fabric or a rubber pipe.

It is necessary to cover vibrating surfaces and equipment with vibration absorbing and damping materials (rubber, special mastics, asbestos, bitumen, Agate plastics, etc.). At the connection points of the mating parts, shock absorbing materials (rubber, cork, cardboard, asbestos, spring shock absorbers) should be used to ensure a snug fit.

Reduce vibration in the vibration source, i.e. in the source of its formation, the following methods are possible: by excluding the impact interaction of the parts from the structure, by replacing the reciprocating movement of the parts by the rotational one, by eliminating the imbalance of rotating parts and machine components.

When working with pneumatic and electric manual machines, vibration occurs, transmitted through the handles and cases to the hands of workers, and sometimes to the legs through the medium being processed, usually when working with rammers and vibrators. To reduce vibration in this case, use handles with vibration damping or automation devices.

Personal protective equipment against vibration is used when other means are ineffective.

Shoes with shock-absorbing soles, handguards with vibration-absorbing elastic gaskets, etc. are used as personal protective equipment against vibration.

Under the influence of intense noise and vibration, fatigue and irritability, poor sleep, headache, weakening of memory, attention and visual acuity occur, which leads to a decrease in labor productivity (on average by 10-15\%) and is often the cause of injuries. Vibration and noise affect the cardiovascular, endocrine and nervous systems, disrupt the coordination of movements. Adaptation to noise is not possible.

\section{Acknowledgements}

The study had no sponsorship. 


\section{References}

1. A.T. Rybak, V.P. Zharov, A.V. Serdyukov, Russian Engineering Research 29(2), 194197 (2009)

2. A. Rybak, O. Lyakhnitskaya, MATEC Web of Conferences 132, 01001 (2017)

3. A.T. Rybak, A.R. Temirkanov, O.V. Lyakhnitskaya, Russian Engineering Research 38(9), 702-704 (2018)

4. A.T. Rybak, A.R. Temirkanov, O.V. Lyakhnitskaya, Russian Engineering Research 38(3), 212-217 (2018)

5. K. Kobzev, S. Shamshura, A. Chukarin, V. Bogdanovich, V. Kasyanov, MATEC Web of Conferences 226, 01022 (2018)

6. K. Kobzev, S. Shamshura, A. Chukarin, A. Buryanovand, V. Kasyanov, MATEC Web of Conferences 226, 01023 (2018)

7. K. Kobzev, A. Chukarin, IOP Conference Series: Earth and Environmental Science 403, 012145 (2019)

8. K.O. Kobzev, E.S. Bozhko, A.V. Mozgovoi, M.D. Molev, N.I. Stuzhenko, IOP Conference Series: Materials Science and Engineering 680, 012014 (2019)

9. K.O. Kobzev, E.S. Bozhko, A.V. Mozgovoi, E.I. Kostromina, L.G. Babenko, IOP Conference Series: Materials Science and Engineering 680, 012013 (2019)

10. N. Kobzeva, V. Terentev, I. Zolotuhina, IOP Conference Series: Materials Science and Engineering 680, 012046 (2019)

11. N. Kobzeva, V. Terentev, I. Zolotuhina, IOP Conference Series: Materials Science and Engineering 680, 012011 (2019)

12. D. Larin, E. Staseva, E. Khetsuriani, IOP Conference Series: Materials Science and Engineering, Collection of materials of the XV International Scientific - Technical Conference Don State Technical University, 012016 (2019)

13. A. Sazonova, O. Kopytenkova, E. Staseva, IOP Conference Series: Materials Science and Engineering 21, 032039 (2018) 